\title{
NON-MARKOVIAN PROCESSES WITH THE SEMIGROUP PROPERTY
}

\section{By William Feller ${ }^{1}$ \\ Princeton University}

1. Introduction. Every $N \times N$ stochastic matrix $P$ defines the transition probabilities of a Markovian process with positive discrete time parameter. Its $n$-step transition probabilities satisfy the Chapman-Kolmogorov, or semigroup, relation $P^{n+m}=P^{n} P^{m}$. We shall show that for $N \geqq 3$ there exist non-Markovian processes with $N$ states whose transition probabilities satisfy the same equation. ${ }^{2}$ All elements of $P$ will equal $N^{-1}$. The process may be chosen strictly stationary. A simple modification leads to non-Markovian processes with continuous time parameter and the semigroup property with $N$ states or a continuum of states.

The triviality of the following example should not obscure the interest of the problem concerning the existence of non-Markovian processes satisfying the Chapman-Kolmogorov equation. As so many other basic problems in probability theory, it has been formulated by P. Lévy who with his usual ingenuity gave the first counter-example to the obvious conjecture.

2. Let $\mathfrak{B}$ be the sample space whose points $\left(x^{(1)}, \cdots, x^{(N)}\right)$ are the random permutations of $(1,2, \cdots, N)$ each carrying probability $1 / N$ ! Let $\Re$ be the set of the $N$ points $\left(x^{(1)}, \cdots, x^{(N)}\right)$ such that $x^{(i)}=\nu$ for all $1 \leqq i \leqq N$ where $\nu$ is a fixed integer $1 \leqq \nu \leqq N$; each point of $\Re$ carries probability $1 / N$. Finally, Let $\subseteq$ be the mixture of $\mathfrak{B}$ and $\Re$ with $\mathfrak{B}$ carrying weight $1-N^{-1}$ and $\Re$ weight $N^{-1}$.

More formally, $\subseteq$ contains the $N !+N$ arrangements $\left(x^{(1)}, x^{(2)}, \cdots, x^{(N)}\right)$ which represent either a premutation of $(1,2, \cdots, N)$ or the $N$-fold repetition of an integer $\nu, 1 \leqq \nu \leqq N$. To each point of the first class we attribute probability $\left(1-N^{-1}\right)(N !)^{-1}$, to each point of the second class probability $N^{-2}$.

Then clearly

$$
P\left\{x^{(i)}=\nu\right\}=N^{-1}, \quad P\left\{x^{(i)}=\nu, \quad x^{(j)}=\mu\right\}=N^{-2}
$$

for all $i \neq j$. Thus all transition probabilities are equal:

$$
P\left\{x^{(i)}=\nu \mid x^{(j)}=\mu\right\}=N^{-1} .
$$

Given, say, that $x^{(1)}=1, x^{(2)}=1$ the probability that $x^{(3)} \neq 1$ is zero, and hence the process is non-Markovian.

3. To extend the process to all integral values of the time parameter consider, in the usual manner, a double infinity of independent repetitions of the described

\footnotetext{
Received February 4, 1959.

${ }^{1}$ Research supported by the Office of Ordnance Research U. S. Army Contract DA-36034-ORD-2001.

2 [Added in proof.] D. Blackwell has pointed out to me that the variables of our process represent a sequence of random variables which are pairwise independent without being mutually independent.
} 
sample space. In other words, we consider the product space $\cdots \subseteq \times \subseteq \times \subseteq \ldots$ with product measure; its points are the doubly infinite sequences $x=\left\{x^{(i)}\right\}$ such that for each integer $r$ the $N$-dimensional block $\left(x^{(r N+1)}, x^{(r N+2)}, \cdots, x^{(r+1) N}\right)$ represents the projection of $x$ onto a coordinate space $\mathfrak{S}$. This represents a nonMarkovian process with the stationary transition probabilities (2). However, the process itself is not stationary in view of the periodicity modulo $N$.

To obtain a stationary process of the same type it suffices to introduce $N$ replicas of our process with time shifts $0,1,2, \cdots, N-1$ and define a new process as their mixture with equal weights.

4. To construct a process of a similar character defined for all $t \geqq 0$ consider the above discrete process and a Poisson process $\{N(t)\}$ with mean $t$ independent of it. Define a new process by

$$
x(t)=x^{(N(t))} .
$$

Its absolute probabilities are given by

$$
P\{x(t)=\nu\}=\sum_{i=0}^{\infty} P\{N(t)=i\} \cdot P\left\{x^{(i)}=\nu\right\}=N^{-1} .
$$

The joint probabilities for $0 \leqq s<t$ are calculated in like manner, but the possibility that $N(t)=N(s)$ makes it necessary to consider separately the cases $\nu=\mu$ and $\nu \neq \mu$. Clearly

$$
\begin{aligned}
P_{\nu \mu}(t) & =P\{x(s+t)=\mu \mid x(s)=\nu\} & \\
& =N^{-1}\left(1-e^{-t}\right), & \text { if } \nu \neq \mu, \\
P_{\nu \nu}(t) & =e^{-t}+N^{-1}\left(1-e^{-t}\right) &
\end{aligned}
$$

and a simple calculation shows that

$$
P_{\nu \mu}(s+t)=\sum_{\lambda=1}^{N} P_{\nu \lambda}(s) P_{\lambda \mu}(t)
$$

even though the process is clearly non-Markovian.

- Finally, one might replace the $N$ states by $N$ intervals and an appropriate motion within them.

\section{REFERENCE}

[1] P. LÉvy, "Exemples de processus pseudo-Markoviens," Comptes Rendus Académie Sciences, Paris, Vol. 228, (1949), pp. 2004-2006. 